"Now move like that fish": Can enactment help learners come to understand dynamic motion presented in photographs and videos?

\author{
Katharina Scheiter ${ }^{\mathrm{a}, \mathrm{b}^{*}}$, Birgit Brucker ${ }^{\mathrm{a}}$, Shaaron Ainsworth ${ }^{\mathrm{c}}$ \\ ${ }^{a}$ Leibniz-Institut für Wissensmedien, Tübingen, Germany \\ ${ }^{\mathrm{b}}$ University of Tübingen, Germany \\ ${ }^{\mathrm{c} S c h o o l ~ o f ~ E d u c a t i o n, ~ U n i v e r s i t y ~ o f ~ N o t t i n g h a m, ~ E n g l a n d ~}$
}

*Correspondence concerning this article should be addressed to Katharina Scheiter, Leibniz-

Institut für Wissensmedien, Schleichstraße 6, 72076 Tuebingen, Germany; email: k.scheiter@iwm-tuebingen.de. 
Running head: Enacting Fish Locomotion

"Now move like that fish": Can enactment help learners come to understand dynamic motion presented in photographs and videos? 


\title{
"Now move like that fish": Can enactment help learners come to understand dynamic motion presented in photographs and videos?
}

\begin{abstract}
Technological advancements offer new possibilities of interacting with learning materials, including the use of gestures and body movements. The present study addressed the question of how using one's body to enact movements whilst learning about them would affect outcomes. 85 participants were shown either sequences of photographs or videos of fishes deploying different locomotion patterns for propulsion. Half of the participants in each visualization condition were prompted to enact the movements whilst learning. During learning, all participants were asked to rate their mental effort; moreover, their enactments were videotaped and later coded with respect to their frequency of occurrence and their congruency with the actual fish locomotion. After the learning phase, students were asked to classify fish based on their locomotion behavior as well as to describe fish showing familiar and unfamiliar locomotion behaviors to assess learning outcomes. Results showed that - independent of visualization format - being asked to enact the fish movements had a positive effect on the students' ability to classify fish as long as the locomotion behavior was neither too easy nor too difficult to be recognized. It did not affect the ability to describe fish movements. The frequency of enactments and their congruency were unrelated to learning outcomes. Taken together, the effects of enactments in this study appear to be limited to certain tasks. Furthermore, they are likely to be due to enhancing engagement in the learning rather than to mechanisms specific to enacting body movements.
\end{abstract}


Keywords: visualization; enactment; gestures; learning about movements

\section{Introduction}

The way people interact with learning technologies is changing. Whilst the keyboard and the mouse may still predominate, there are increasing applications which ask learners to write or draw with digital pens, feel forces in their fingers via haptic joysticks or manipulate physical objects to change their digital representation (tangibles). In particular, there are many more applications that allow people to control them with their hand and body movements, and such gesture-based learning environments are increasing rapidly (Sheu \& Chen, 2014). This evolution in the technological landscape goes hand in hand with our growing knowledge of how learning with our bodies shapes our understanding (Alibali \& Nathan, 2012; Glenberg, 2010; GoldinMeadow\& Wagner, 2005) and can be utilized to help people learn (Lindgren \& JohnsonGlenberg, 2013). However, as yet there is more excitement than there are studies that have delimited the particular activities where embodiment supports learning with technology. Thus, this paper reports on a study that asked people to physically enact with their bodies visual material they were studying. It compares learning from both static and dynamic visualizations as it has been argued that such an approach may be particularly useful with animations and video (de Koning \& Tabbers, 2011). Moreover, by measuring the types of body actions learners make and relating these to the outcomes of learning, we hoped to explore not just whether enactment helps learning from visual materials but why it might (or might not) be helpful in this case.

\subsection{Enactment as a learning strategy}

It is now well established that learning from visual materials is more effective if students are encouraged to study them using an appropriate learning strategy (e.g., for overarching reviews 
see Chi, 2009; Dunlosky, Rawson, Marsk, Nathan, \& Willingham, 2013; Renk1 \& Scheiter, 2017). For example, students' learning is enhanced if they engage in inferential processes brought about when they self-explain diagrams (e.g., Author C, 2003; Cromley et al., 2013). Similarly, learning from animations is increased if students are taught (and then apply) strategies that encourage them to select, organise, transform and integrate the information depicted (Kombartzky, Ploetzner, Schlag, \& Metz, 2010) or when they draw key aspects of the animation (Mason, Lowe, \& Tornatora, 2013). When learners do not apply such strategies, extensive research suggests that far too frequently people can fail to attend to important information in the visualizations, either skipping them altogether (e.g., Hegarty \& Just, 1993; Schmidt-Weigand, Kohnert, \& Glowalla, 2010); attending to superficial salient details, not relevant information (e.g., Hegarty, Canham, \& Fabrikant, 2010; Lowe, 2004) or failing to integrate them with other representations (e.g., Author C, 2006; Seufert, 2003). In this study of learning from complex visual materials, we asked whether using your body in a meaningful way (enactment) could also be as a successful learning strategy. We use the term 'enactment' as it encompasses many ways of using one's body to express and development understanding rather than 'gesturing'as this is often defined to include only hand and or occasionally head movements ( e.g. "Gestures are spontaneous hand movements that accompany speech", Novack \& Goldin-Meadow, 2017, p. 652). The exception to this terminological approach is when we describe the research of others and hence use the terms that authors themselves used.

Interest in embodied learning is increasing as research has found that the enacting ${ }^{1}$ of knowledge and concepts through bodily activity can support learning. For example, when people gesture as they solve problems and reason, their understanding is enhanced (e.g., GoldinMeadow \& Wagner, 2005; Stieff, Lira, \& Scopelitis, 2016). Chu and Kita (2011) asked people to 
solve $3 \mathrm{~d}$ mental rotation tasks and found that those who spontaneously gestured solved difficult problems more accurately. Moreover, this is unlikely to be epiphenomenal as, in a follow-up study, when participants were encouraged to gesture, they solved these problems more accurately than people who were not permitted to gesture. Furthermore, even when the participants were prohibited from gesturing in a subsequent problem-solving phase this advantage was maintained. These benefits of gesturing are not only found in laboratory tasks. Cook, Mitchell, and GoldinMeadow (2008) found that requiring children to gesture whilst they solved mathematics problems involving grouping was associated with better performance four weeks later. Gesturing as a learning strategy can also be trained. For example, Stieff et al. (2016) taught students gestures, which they then practiced, that represented the spatial relationships depicted in diagrams of molecular representations. These students performed better than those who studied alone or who observed a teacher who gestured these relationships. They did equally well to those taught with concrete models and better in an exam when concrete models were not available.

Research has also addressed whether enactment and gesturing can also help learning from visual materials. This research can be classified in terms of how human movement is employed in the studies. The majority of the research has explored whether the observation of included gestures or representations of hands in learning materials facilitates students' understanding (e.g., de Koning \& Tabbers, 2011; Pi, Hong, \& Yang, 2017; Schwan \& Riempp, 2004). This research typically does find a benefit; although not invariably so (see Castro-Alonso, Ayres, \& Paas, 2014 for a counterexample). However, we are interested in whether asking learners themselves to move facilitates learning and here the research is decidedly mixed. Post, van Gog, Paas and Zwaan (2013) compared young children learning about grammar from animations when they either saw an animation or saw the animations which also included procedural information about 
grammar rules in gestures which the children had to mimic. Contrary to their expectations, there was no overall benefit of gesturing and indeed for children who began the study with lower language skills this activity even harmed their performance on later tests. De Koning and Tabbers (2013) compared participants learning from an animation of the lightening cycle when they either had to watch the animation, watch the animation where important movements were cued by a pointing with a photograph of a human hand or watch the animation where important movements were cued by a pointing arrow that they also needed to gesture towards. Again, gesturing along with the arrow was no more successful than simply watching the animation (and not as successful as watching a human hand). The authors speculated that any potential benefits of gesturing may have been obscured by the unusual nature of this constrained gesturing activity.

In contrast, Macken and Ginns (2014) do find that gesturing activities including tracing over diagrams and between text and diagrams enhanced learning about the human heart. Similar gesturing and tracing activities were used by Hu, Ginns and Bobis (2015) who also found an advantage for these activities in the context of geometry worked-out examples. Moreover, Lajevardi, Narang, Marcus, and Ayres (2017) do find a benefit of making gestures whilst studying visual materials. In their studies, learners were studying how to write unfamiliar characters (in Mandarin and Persian) and some additionally had to mimic the writing actions they observed. However, half the participants observed animations whereas the others observed static materials which showed the keyframes but no movements. Their results indicated that gesturing was beneficial in the static but not animated conditions. Existing research is therefore mixed, suggesting that although learners can benefit from enactment, there is only a limited number of situations where this approach has been found to enhance learning from visual materials. 
Although this may initially seem disappointing, it is perhaps not unexpected as research has shown that even those learning strategies that have utility across different study materials, such as self-explanation or drawing have been found to have boundary conditions and situations where they do not help learning (e.g., Ploetzner \& Fillisch, 2017). We suggest that one way of clarifying this is to consider the mechanisms by which enactment may help learning; considering both fairly general mechanisms as well as very specific ones.

General mechanisms by which enactment could support learning from visual materials include increasing learners' motivation to study. Being asked to gesture and enact is, in our experience, a rather unusual approach to support learning and one that students will rarely encounter in formal school. Consequently, being encouraged to do so in experimental situations may serve to enhance motivation in general due to its novel nature. Similarly general are the benefits that might come from increasing participants' cognitive and physiological arousal in line with predictions from arousal theory (e.g., Yerkes \& Dodson, 1908; Lambourne \& Tomporowski, 2010). Potentially, increasing one's physical activity to greater levels might enhance cognitive performance, especially as such activity is unlikely at maximal levels. These types of benefit would be expected to accrue irrespective of how participants respond and the nature of the learning materials.

More specific benefits of enactment, however, would depend upon the nature of learners' enactment and how this aligns with the form and content of the studied material. One argument for the benefits of gesturing to learn is that people are particularly attuned to learn about human movement through bodily experiences, either their own or others (de Koning, \& Tabbers, 2011). This, it is suggested, explains why people are particularly good at learning by watching animations of complex motor and procedural skills such as people tying knots (Schwan \& 
Riempp, 2004) or building with construction toys (Castro-Alonso, Ayres, \& Paas, 2015). This argument often recruits an explanation based upon activation of the human mirror neuron system (e.g., Ayres, Marcus, Chan, \& Qian, 2009). For example, Author B (2015) used functional NearInfrared Spectroscopy (fNIRS) and showed increased activation in the inferior-frontal cortex of participants low in visual-spatial abilities when they watched instructional gestures. If human movement is not shown but people need to enact something dynamic they have seen, it would be predicted that the success of this approach would depend upon whether these movements are ones they can successfully embody. Consequently, this predicts that the content of the studied material will have a strong influence on the likelihood of enactment being a successful way to learn.

Enactment may be particularly helpful when learning with visual materials and especially animations as previous research points out these can both "underwhelm" or "overwhelm" learners (Lowe, 2003). When visual materials underwhelm, learners pay insufficient attention to them, focus on surface and superficial features and process them only shallowly (e.g., Harp \& Mayer, 1997; Sanchez \& Wiley, 2006). This tendency is particularly marked with animations (Lowe, 2003). Consequently, being required to enact studied visual materials with one's own body may focus learner's attention on the important aspects of the visual materials, in similar ways to other constructive strategies such as drawing (Wiley, 2019). Alternatively, animations, are notorious for the way that their transient nature burdens learners' limited working memory capacity (Lowe, 2003). It is argued that using one's body to represent the contents of animation can offload cognition from limited working memory capacity onto one's hands, hence freeing up cognitive resources for more extensive processing of the animation (e.g., Lajevardi, Narang, 
Marcus, \& Ayres, 2017; de Koning \& Tabbers, 2011, 2013; Pouw, de Nooijer, van Gog, Zwaan, \& Paas, 2014).

A final level of explanation concerns what learners specifically do with their bodies: that is, whether their gestures and enactments are representational and appropriate. Kita, Alibali and Chu (2017, p. 245) suggest that gestures can help learning when they act as abstracted representational motor actions that depict "action, motion, or shape, or that indicate location or trajectory”. Similarly, Novack and Goldin-Meadow (2017) argue that as representational gestures abstract away from the specifics of the situation to highlight its key properties this can support understanding, retention, and generalization. In this way, they can become, as Pouw et al (2014) argue, epistemic actions that reveal by their representational properties aspects of a situation or phenomenon that might otherwise not be made visible. This explains why some representational gestures are more effective than others (Goldin-Meadow, Cook, \& Mitchell, 2009). These movements can be relatively simple such as the V-shaped gestures to components of mathematics problems (e.g., Goldin-Meadow et al., 2009) or they can be more complex roleplays such as when children enact the orbit of the moon around the earth or the movement of water molecules in ice (Tytler, Prain, Hubber, \& Waldrip, 2013). Moreover, this body-based knowledge may result not only in an extra sensory code in memory (Engelkamp \& Zimmer, 1984) but it may also result in new conceptual metaphors (e.g., Lakoff \& Johnson, 1980; McNeil, 1992) that help learners make sense of a situation in a new way. For this to be successful though, these actions must be congruent with the core conceptual structures to be learned (Lindgren \& Johnson-Glenberg, 2013; Pouw et al, 2014).

In elucidating these arguments and presenting them as separate aspects of enactment we do not mean to imply that bodily action serves only one purpose at a time; for example, learners 
may be motivated by the physical activity involved in making new representational gestures that embody key aspects of the studied material. Nonetheless, in designing our study we paid close attention to these different mechanisms so that we could manipulate variables that may impact upon them and measured both the processes and outcomes of enactment-based learning in an attempt to distinguish whether enactment had more general or specific effects in this case.

\subsection{Hypotheses}

The material learners studied in this experiment concerned fish locomotion: that is, participants studied visual representations of fish movements in order to identify different patterns (for previous studies with same learning task and similar materials see Authors A \& B, 2011, 2012, 2013, 2014). Recognizing such locomotion patterns is non-trivial as learners must consider both temporal aspects and spatial aspects that form the basis of species classification (Videler, 1993). Thus, the content of the studied material is about bodily movement but, similarly to the classic example where people fail to understand horse locomotion, participants are required to understand bodies very different from their own. Consequently, if enactment does help learning the explanation cannot rest on those mechanisms proposed for understanding human movement (e.g., de Koning \& Tabbers, 2011) but instead still could help learning through the other general and specific mechanisms we articulated above.

H1: Participants who enact fish locomotion in their own bodies will have enhanced learning outcomes compared to those who do not.

We used different forms of visual material, not because we were interested in whether dynamic or static study materials help people learn best as many other studies have addressed that issue (e.g., Höffler \& Leutner, 2007; Berney \& Bétrancourt, 2016). We did so we could 
specifically test whether dynamic and static materials benefited most from enactment. Given the mixed results reviewed above, we formed two alternative hypotheses concerning how enactment would influence learning from the different forms of visual materials.

H2a: Participants who watch videos whilst enacting will have enhanced learning outcomes compared to those who view photographs whilst enacting.

We made this prediction on the basis that videos are transient representations. Hence participants will benefit from offloading their cognition onto their hands (consequently we take self-reported measures of mental effort) and in attempting to emulate the motion they see they may place closer attention to the video and process it less superficially. Previous research which found a benefit for gesturing from a video (Lajevardi et al., 2017) also indicated that this benefit only manifested when the materials studied were at least moderately complex and therefore we also expected that this may be true in our materials.

H2b: Participants who view photographs whilst enacting will have enhanced learning outcomes compared to those who watch videos whilst enacting.

In this case, participants viewing photographs do not have access to the full dynamic information provided by the videos and so by using their bodies as an active strategy, this could compensate for this missing information.

In order to test whether the benefits of enactment were (in part at least) due to learners forming schematic representations of the fish locomotion in their own bodies, we also videoed the participants whilst they studied the material. The enactments they made were coded in terms of their presence or absence and their congruency (see section 2.3). Consequently, we made the following predictions. 
H3: As video provides a fuller indication of the fish movements, participants in the video condition will make more congruent enactments than those viewing still photographs.

H4: Participants who make congruent enactments will learn more than those who make less congruent ones.

\section{Method}

\subsection{Participants and design}

Eighty-five students (with different majors from a German University) participated in the study for either payment $(16 €)$ or course credit. Two participants had to be excluded from data analyses: One participant did not comply with the instruction and did not perform any gestures when being prompted to do so. The other participant was a Major in Biology and possessed too much background knowledge regarding the domain of the study. This left 83 participants (59 female; $\mathrm{M}=24.16$ years, $\mathrm{SD}=4.24$ ). Given that we were interested in how enactments would enhance learning from different visual formats (i.e., static versus dynamic materials), these two factors were varied experimentally in a $2 \times 2$ between-subjects design. That is, students learned from either photographs (static) or videos (dynamic), thereby varying the visual format of instruction. Furthermore, half of the students were prompted to perform enactments of what they observed in the visuals, while the other half received no enactment prompts (enactment prompt). The participants were randomly assigned to one of the four conditions. There were 22 participants in the condition without enactment instruction and photographs, 21 participants in the condition without enactment prompt and videos, and 20 participants in the two remaining conditions. 


\subsection{Materials}

The study materials consisted of six videos, one per fish, whereby each fish deployed a different locomotion behavior (i.e., a pattern of moving forward). The movement patterns varied with respect to the body parts a particular fish uses to generate movement (i.e., whole body, pectoral fins or dorsal and anal fins) and the way in which it moves these body parts (i.e., in a wave-like, undulating or paddle-like, oscillating manner): (1) anguilliform: undulation of the body as a whole with constant amplitudes (e.g., eel); (2) subcarangiform: undulation of the body as a whole with decreasing amplitudes (e.g., trout); (3) rajiform: undulation of enlarged pectoral fins, mostly in addition with oscillating movements of these fins (e.g., ray); (4) balisitiform: undulation of the dorsal and anal fins (e.g., triggerfish); 5. tetraodontiform: oscillation of the dorsal and the anal fins, as well as sometimes undulation of the pectoral fins (e.g., puffer fish); and 6. labriform: oscillation of the pectoral fins (e.g., wrasse). One of the major challenges in identifying these locomotion patterns is that fish may deploy a variety of additional movements, for instance, in order to navigate, which may easily be confused with these characteristic movements pertaining to propulsion.

Depending on the condition, there was either a single video of each fish or a series of sequentially presented photographs. The videos were non-interactive underwater videos showing a fish performing one complete movement cycle. The photographs consisted of stills extracted from the videos (see Figure 1 for an example of the tetraodontiform movement pattern), which corresponded to the key states of the movement as determined by two domain experts (cf. Authors A \& B, 2012). For each movement pattern, nine stills were chosen. Each still was presented for three seconds and then automatically replaced by the next picture in the sequence. 
For learning, the visualizations of all six fish were shown once (trial 1) and then a second time (trial 2; see procedure for details).

\subsection{Measures}

As control variables, we assessed participants' spatial abilities, their verbal abilities, and their familiarity with related tasks and contents. Moreover, we asked the participants whether they had received training in either dancing or acting since this might influence their propensity to use their body as a way of expressing themselves. There were no differences between conditions regarding the number of participants who had received either type of training. As dependent variables, we assessed learning outcomes by means of a movement pattern classification task (recognition) with a range of difficulty and two open-ended near and far transfer written description tasks. Furthermore, self-reported mental effort during learning was registered. Finally, the body movements that students produced were coded. Each of these is explained in more detail below.

Spatial ability. Participants' spatial ability was assessed with a shortened paper-pencil version of the Paper Folding Test (PFT, Ekstrom, French, Harman, \& Dermen, 1976). It consisted of 10 multiple-choice items. Each item showed a square piece of paper being folded and then punched with a pencil as well as five possible variants of that same paper when the paper is completely unfolded. Participants were asked to determine which of the five versions correctly represented the folded paper stimulus. They had three minutes to work on the PFT. For each correct answer, a participant received one point and for each incorrect answer, one point was deducted, yielding a minimum of -10 points and a maximum of +10 points. 
Verbal abilities. Participants' language skills were tested with the MehrfachwahlWortschatz-Intelligenz-Test (MWT-B, Lehrl, 2005). The MWT-B consists of 37 items, whereby for each item there are four non-words (meaningless letter combinations) and one meaningful word. The task was to identify the meaningful word. There were no time limits for this test. For each correct answer, a participant received one point (max. 37 points).

Familiarity with the domain. The questionnaire to assess participants' familiarity with the domain assessed the number of courses taken in Biology and Physics (details of participants' school education), experiences with the marine world (e.g., diving, snorkeling, swimming, rowing, and/or owning an aquarium), interest in related topics (e.g., biology, zoology, physics, aircraft construction, and/or shipbuilding) and media use (e.g., documentaries and/or books on fish or sea life). Participants could achieve a total amount of 25 points in this questionnaire, which served only to identify students in the unlikely event that they had expertise in this otherwise rather exotic domain.

Learning outcomes. Recognition was assessed in a movement pattern classification test. In this test, participants saw 28 underwater videos of fish that displayed the same movements as the previously studied fish but that differed their visual appearance (i.e., shape and color). There were four to six videos for each to-be-learned movement pattern. Students had to classify the movement pattern of each fish by assigning it to the corresponding movement pattern from the learning phase. This was done non-verbally: for each movement pattern from the learning phase, one still was extracted from the videos to represent the respective movement pattern. Thus, students did not have to be able to name the movement patterns; rather, they could classify a test item simply by assigning it to the representative photograph. 
The 28 items were chosen so that they represented different levels of task difficulty. In easy items (13 items) the depicted fish showed the locomotion pattern for propulsion continuously, and the fish showed no other movements. Data for four of these items showed too little variance as almost all students recognized the fish correctly (i.e., ceiling effect); therefore, these items had to be excluded from the analysis. In the medium difficulty items ( 7 items), the depicted fish showed the relevant locomotion pattern continuously, but they also performed some movements that were similar to another locomotion pattern. In difficult items ( 8 items) the depicted fish either showed the pattern relevant for propulsion continuously, but contained additional movements similar to at least two other locomotion patterns, or the depicted fish did not show the relevant locomotion pattern for propulsion continuously or did not show it in a salient manner. Participants received one point for each correct answer, yielding 9, 7, and 8 points for easy, medium, and difficult items, respectively, which were transformed into ratios for easier interpretation.

Additionally, learning outcomes in terms of near and far transfer were assessed with written open description tasks. In the near transfer test, participants were shown videos of four novel fish that performed the same continuous propulsions as the fish seen during learning. In the far transfer test, participants were shown videos of four marine animals (three fishes and a cuttle) that showed different movement behaviors to the ones from the learning phase. In both tasks, participants were asked to describe the fish's movements as precisely as possible and received points whenever they recognized which body part is important for generating propulsion and which body part is important for navigation (1 point for each item). Additionally, they received points whenever they described correctly how these body parts moved. For 3 out of 4 items in each test, this description required mentioning one aspect (1 point for each item), whereas in one 
item it required mentioning two aspects ( 2 points). Thus, there was a maximum score of 9 for both transfer tests. The total score was converted into a ratio for easier interpretation.

Mental effort. Mental effort was assessed during learning separately for each of the six tobe-learned locomotion patterns. Thus, participants were asked to rate how much effort they had invested during learning on a 7-point Likert scale from "none at all" to "very much" after each movement pattern was presented during the first learning trial.

Enactment coding. Participants were videotaped during the whole experiment with two cameras positioned at different viewing angles so that their body movements during learning could be coded. First, one of the authors of this paper determined for each of the 6 movement patterns shown during learning whether students had performed enactments and if so, whether they appeared related to the fish movement (relevant) or unrelated (e.g., communicative or other gestures). The number of fishes for which students performed a movement related to the locomotion of the fish at least once (that is, either during trial 1 or 2 or during both trials) was used as a dependent measure. In cases where a participant displayed more than one enactment, the two coders determined which was the dominant one and would thus be chosen for further analysis. In cases of disagreement, a consensus was achieved by discussion. For the chosen enactments, coders decided if the enactment was congruent with the fish movement $(0=$ not at all, $1=$ some, 2 = close match).

\subsection{Procedure}

Participants were tested in single sessions. To allow them to use their whole body, while at the same giving them the opportunity to sit during the experiment, the following set-up was chosen. The computer on which the materials were displayed was placed on a table whose height was 
adjusted so that a person could easily stand in front of it while watching the screen. Placed in front of the table was a bar stool that allowed maximum mobility with the upper body part whilst sitting on it. Also, students could easily move it away if they preferred to gesture while standing. Two video cameras were positioned to the left and at the right side of the table, recording the participants from the front and each side.

Once students were comfortable with this arrangement, they were first given a short computer-based written overview on the experiment and an instruction that they were to learn as much as possible about the movement patterns deployed by different fish so that they later would be able to recognize and describe these patterns as accurately as possible. Participants were furthermore informed that they could watch the videos/photographs sequences for as long as they liked and that each video/photograph sequence would restart automatically until they pressed the space to continue to the next movement pattern. In addition, participants in the conditions with enactment prompt received the following instruction: "Whilst you watch the visualizations, please use your hands and body to gesture in a way that you think will help you to better understand the fish's movements. This might sound strange; however, previous research has shown that gestures deployed in such a way may improve learning and that they do so particularly if they are matched to what is to-be-learned"1.

The instructions were followed by the learning phase, in which the visualizations for the six movement patterns were presented at the center of the computer screen. After each presentation of the six movement patterns, participants were asked to rate their mental effort concerning it. These sequences of six movements patterns were then presented for a second time. After the learning phase, participants first performed the near transfer task. They were instructed to describe the movements of the fish shown in the four videos as precisely as possible. 


\begin{abstract}
Afterward, participants performed the classification task. They were instructed to classify the movement patterns depicted in this task by comparing them with the movement patterns of the learning phase. Then, participants performed the far transfer task. They were again instructed to describe the movements of the fish shown in the four videos as precisely as possible. No time restrictions were giving for any of the learning outcome tests. Finally, participants answered the PFT and the MWT-B. Then they were debriefed and thanked for their participation. A single session took approximately 90 minutes.
\end{abstract}

\title{
3. Results
}

The first step in the analysis was to check whether the conditions differed regarding the students' verbal and spatial abilities and their domain familiarity (Table 1). A multivariate ANOVA with visual format (photographs vs. video) and enactment prompt (with vs. without) as factors revealed no effects of either factor and no interaction $\left(\right.$ all $\left._{\mathrm{s}}<1\right)$. Correlational analyses between students' entry characteristics and overall performance in the description task and the classification task were performed. There were no significant correlations between the students' verbal ability score and performance (description task: $r=.04 ; p=.74$; classification task: $r=$ $.005 ; p=.96$ ) or between domain familiarity and performance (description task: $r=.05 ; \mathrm{p}=.65$; classification task: $r=-.17 ; p=.13$ ). In line with the literature (cf. Höffler, 2010), spatial ability as measured by the PFT, was positively correlated with performance (description task: $r=.32 ; p$ $=.004$; classification task: $r=.37 ; p=.001$ ), which is why it was considered as a covariate ${ }^{2}$ in the subsequent analyses.

\subsection{Mental effort, enactment quantity and congruency}


Students' averaged ratings regarding their experienced mental effort were analyzed with a two-factorial ANCOVA with visual format and enactment prompt and spatial ability as covariate. Means and standard deviations are displayed in Table 2. There were no significant effects (enactment prompt: $F(1,78)=1.33, M S E=1.82, p=.25, \eta^{2}=.02$; visual format: $F(1,78)$ $=3.06, M S E=1.82, p=.08, \eta^{2}=.04$; enactment prompt $x$ visual format: $\mathrm{F}<1$ : spatial ability: $\left.F(1,78)=2.86, M S E=1.82, p=.095, \eta^{2}=.04\right)$. Thus, neither the format of the studied visual material or the prompt to enact seemed to affect participants' experiences of effort.

Furthermore, the number of fishes where participants performed a relevant body movement at least once was determined (Table 2). In the prompted conditions, this coding was used to identify the single person who had not complied with the prompt, who was then excluded from all analyses. However, in the conditions without the prompt, 20 out of 43 participants performed at least one enactment despite not being told to do so. We decided against removing these participants as this would not only have drastically reduced our sample size, it would also have yielded a non-representative sample.

The number of fishes with enactment was analyzed using a two-factorial ANCOVA with visual format and enactment prompt as factors, and spatial ability as covariate. As expected, the prompt increased the number of enactments (with prompt: $M=5.92, S E=0.62$; without prompt: $M=2.15, S E=0.30), F(1,78)=74.37, M S E=3.95, p<.001, \eta^{2}=.49$. Hence, our experimental manipulation had been successful in that those students who had been prompted to enact the observed movements performed enactments more frequently. Visual format had no significant effect on the number of enactments performed nor was there an interaction between visual format and enactment prompt (both $F_{\mathrm{s}}<1$ ). Also spatial ability had no effect on the number of enactments $F(1,78)=1.57, M S E=3.95, p=.21, \eta^{2}=.02$. 
In the next step, we analyzed the mean correspondence between the participants movements and the actual movement displayed by the fish as a measure of congruency using the same analysis as above. This variable was of interest, because we had assumed that videos would yield more congruous enactments than stills (Hypothesis 3) and that students performing more congruous enactments would also show better learning (cf. Hypothesis 4). Note that in the conditions without prompts, there were only 20 students who performed spontaneous enactments; thus, the sample size in the not prompted conditions is reduced to $n=10$ for each visual format condition. There were no main effects of spatial ability, $F(1,55)=1.16, M S E=$ $0.97, p=.29, \eta^{2}=.02$, visual format $(F<1)$, or enactment prompt, $F(1,55)=1.12, M S E=0.97, p$ $=.30, \eta^{2}=.02$. However, there was a significant interaction between visual format and enactment prompt, $F(1,55)=6.05, M S E=0.97, p=.02, \eta^{2}=.10$. Bonferroni-adjusted posthoc comparisons revealed that in the conditions without prompts, visual format had no effect on congruency (photographs: $M=1.31, S E=0.10$; video: $M=1.17, S E=0.10 ; p=.34$ ). In prompted conditions, however, the enactments were more congruent when based on videos $(M=1.47, S E=0.07)$ than when based on sequences of photographs $(M=1.19, S E=0.07 ; p=.006)$. Hence, in line with Hypothesis 3, videos yielded a more comprehensive mental representation of the observed movements that was expressed in the congruency of the enactments performed by students.

To summarize, even though those students who were prompted to enact while watching the visualizations did so to a greater extent than those who were not instructed, in the latter condition almost $50 \%$ of students did so nevertheless. There were no differences between spontaneous and prompted enactments regarding their quality; however, enactments corresponded best with the fish movements when students were prompted to perform them based on videos. Thus, if congruency of enactments mattered for learning (as assumed in Hypothesis 
4), then we would expect students in the video (compared to the photograph) conditions to show better learning outcomes when performing enactments.

\subsection{Learning outcomes}

Learning outcome measures referred to the students' performance in the movement classification task and in the description task (Table 3).

The students' performance in the movement classification task was analyzed using a twofactorial repeated-measures ANCOVA with visual format and enactment prompt as factors, task difficulty (low, medium, high) representing the repeated measure and spatial ability as the covariate.

There was a main effect of task difficulty, $F(2,156)=51.39, M S E=0.02, p<.001, \eta^{2}=$ .40 , suggesting that tasks classified as easy were indeed solved at a much higher rate $(M=.93$, $S E=.01)$ than tasks classified as either being of medium $(M=.68, \mathrm{SE}=.02, p<.001)$ or high difficulty $(M=.68, \mathrm{SE}=.02, p<.001)$, with the latter not differing from each other ${ }^{3}$. There were no main effects of visual format, $F<1$, or enactment prompt, $F(1,78)=2.87, M S E=0.05, p=$ $.09, \eta^{2}=.04$ nor was there an interaction among the two factors, $F<1$. However, task difficulty interacted with prompt condition, $F(2,156)=3.27, M S E=0.02, p=.04, \eta^{2}=.04$. The Bonferroni-adjusted posthoc analyses revealed that there were no effects of being prompted to enact on easy tasks (without prompts: $M=.93, S E=.02$, with prompts: $M=.93, S E=.02$ ) or difficult tasks (without prompts: $M=.66, S E=.03$, with prompts: $M=.70, S E=.03$ ). However, for classification tasks of medium difficulty, students performed better after having received enactment prompts $(M=.73, S E=.03)$ compared with the no-prompt conditions $(M=.63, S E=$ $.03, p=.03)$. There was no interaction between visual format and task difficulty, $F(1,78)=2.51$, $M S E=0.02, p=.08, \eta^{2}=.03$, and no three-way interaction, $F<1$. Finally, spatial ability had a 
strong positive impact on the performance in the classification task, $F(1,78)=12.53, M S E=$ $0.05, p=.001, \eta^{2}=.14$. Accordingly, as expected in Hypothesis 1, being prompted to perform enactments did improve learning outcomes as assessed with medium-difficulty classification tasks. In contrast to the two conflicting Hypotheses $2 \mathrm{a}$ and $2 \mathrm{~b}$, however, these effects of enactment were independent of the visual format.

Performance in the description task was analyzed with a two-factorial ANCOVA with visual format and enactment prompt as factors, and spatial ability as the covariate. It showed no effects of enactment prompt and no interaction of enactment prompt and visual format, both $F_{\mathrm{s}}<$

1. However, performance was affected by visual format, $F(1,78)=6.63, M S E=0.03, p=.01, \eta^{2}$ $=.08$, in that learners with a sequence of photographs $(M=0.70, S E=0.03)$ performed better than those learning with videos $(M=0.61, S E=0.03)$. Again, spatial ability had a strong positive influence on the performance in the description task, $F(1,78)=8.66, M S E=0.03, p=.004, \eta^{2}=$ .10. Accordingly, positive effects of enactment were limited to classification tasks.

\subsection{Relationships between enactments and learning outcomes}

In a final step, we analyzed the relationship between quantity and quality of the participants' body movements and their learning outcomes on the medium difficulty classification task. This was done by performing a regression analysis within the conditions with the enactment prompts using the number of performed enactments and their correspondence with the fish movement as predictors. The overall regression model was not significant, $\mathrm{F}<1$. Thus, the present results do not confirm Hypothesis 4 according to which more congruent enactments had been expected to yield better learning outcomes.

\section{Summary and Discussion}


This study was developed to explore whether enactment supported learning about nonhuman movement from different forms of visual materials and if it did so, to attempt to establish which of the many different explanations for enactment might be implicated.

We predicted being prompted to enact would increase learning outcomes (H1) and this hypothesis did receive partial support from the findings as classification outcomes on medium difficulty tasks were enhanced if learners enacted. However, this was the only measure to show such a benefit as classification was not improved for easy or difficult classification items and the description task. This finding is similar to that reported by Lajevardi et al. (2017) who also found that tasks with easy movements did not benefit from enactment. It also suggests that enactment (at least in this domain) will not result in richer verbal explanations as there was no effect of enactment on the description task. This suggests only limited benefits for enactment. Notably, these findings on enactment - where one uses the whole body to perform observed movements do not necessarily contradict earlier findings regarding the benefits of gestures (limited to hands and heads) or tracing when learning from visual materials (e.g., Macken \& Ginns, 2014; Hu et al., 2015; Lajevardi et al., 2017). Rather, they suggest that benefits of using one's body may be strongly dependent on the type of movements performed relative to the task that needs to be accomplished. For instance, tracing geometric figures is a tightly controlled activity with the fingers and hands where the movement is constrained by the presented figure. In contrast, in the present study students were free to choose how to enact the fishes' movement using single parts (e.g., their hands) up to their whole body. Future studies hence need to address the task specificity of beneficial effects of using one's body for learning. 
Moreover, the interpretation of enactment effects in the present study is complicated by the fact that whilst we had expected participants to be able to enact when prompted (which all but one did), we had not anticipated so many spontaneous enactments; nearly half of the nonprompted participants performed at least one such body motion, which was not influenced by whether they saw static or dynamic materials. This was not expected as earlier studies with the same materials had not reported seeing spontaneous enactment. Given the wide population sampled from we do not propose the explanation here is program bleed, instead, we speculate that the one key difference between this study and earlier ones with the same material was that the participants were not seated in front of a desk in an office style chair. And their enactments often involved using their bodies in ways that would not be easy in such a seated position.

Moreover, this suggests that many participants intuitively felt they would benefit from enactment in this visually complex learning situation. This argument is support by Eielts et al (2018) who found that spontaneous gesturing in increased when tasks demands increased. Consequently, it seems probable that this spontaneous enactment served to reduce the predicted difference in learning outcomes between the conditions. Therefore, a follow-up study could implement an additional condition where enactment is not permitted as seen in studies such as Chu and Kita (2011) and Eielts et al (2018) although this will now add an additional load of inhibiting a presumably valued way of interacting with the material.

Earlier work had found mixed results concerning whether learning with videos or photographs would most benefit from enactment and consequently, we could not made alternative predictions for our second hypothesis (Hypothesis 2a and 2b; de Koning \& Tabbers, 2013; Post et al., 2013; Lajevardi et al., 2017). We found no interaction between the form of the materials and the enactment condition on learning outcomes suggesting that the benefits of 
enactment on classification tasks were equally experienced with both visual materials. The process measures we analyzed to try and deliminate different mechanisms for enactment effects on learning provide further insight into these results.

One explanation that had previously been proposed for the benefits of enactment on learning from animation, is that it reduces the cognitive demands on processing the animation (e.g., Lajevardi et al., 2017; Pouw et al, 2014); however, participants' mental effort scores were not affected by enactment prompts. Consequently, we think this explanation can be ruled out for the present materials. A second explanation considered was that enactment would compensate for the missing information in photographs as participants would generate this information in their own motor movements. Again there does not seem to be evidence for this account either as there are no differences in learning outcomes or mental effort between people who were prompted to enact whilst viewing photographs and those who did not. There also was no difference in spontaneous enactment by condition. However, we did find as predicted by H3 that participants produced more congruent enactments (i.e., they produced body movements that more closely resembled the fish movements that they observed) when they studied video materials rather than static pictures. However, we did not find that congruency predicted learning outcomes and so cannot find support for $\mathrm{H} 4$ that congruent enactments will be most beneficial. It may be that fish bodies and human bodies are sufficiently dissimilar that schematically representing the movement you observe (or infer) is not helpful and does not activate the human mirror neuron system (e.g., Ayres et al., 2009; Brucker et al., 2015). Consequently, the (limited) benefits of enactment for these materials appear most plausibly to come from fairly general explanations of the advantages of enactment - that it is a motivating way to engage in studying materials and that it may increase cognitive arousal. Additionally, participants may have studied 
the materials more carefully if they needed to enact them. This material must be as neither too easy (so that this additional work is not necessary) nor too difficult (so that learners do not benefit from it) to be recognized. Subsequent studies could adopt additional process measures (such as motivational questionnaires or eye tracking) to more directly test this explanation or imaging techniques such as fNIRS.

A limitation of the present study pertains to the relatively low number of participants, which can be argued to occlude possible effects of enactments. However, similar studies in the field have used comparable sample sizes (e.g., Macken \& Ginns, 2014, had 42 students randomly assigned to two experimental conditions). Nevertheless, future studies should attempt to replicate the present findings using larger samples to generate more robust findings.

In conclusion, this study has demonstrated that there is only limited benefit from enacting non-human movements in participants' own bodies. At this point, there are still many unanswered questions concerning when and how enactment supports visual learning and additional studies are still required. However, we hope that we have responded to Lindgren and Johnson-Glenberg's (2013) call for studies that look beyond the effects of an embodied approach to technologically mediated learning to trying to understand what the mechanisms underlying any effect might be and finding when embodiment may also not help learning.

\section{References}

Alibali, M. W., \& Nathan, M. J. (2012). Embodiment in mathematics teaching and learning: Evidence from learners' and teachers' gestures. Journal of the Learning Sciences, 21, $247-$ 286. https://doi.org/10.1080/10508406.2011.611446

Authors A \& B 2011

Authors A \& B 2012

Authors A \& B 2013 
Authors A \& B 2014

Author B 2015

Author C 2003

Author C 2006

Ayres, P., Marcus, N., Chan, C., \& Qian, N. (2009). Learning hand manipulative tasks: When instructional animations are superior to equivalent static representations. Computers in Human Behavior, 25, 348-353. https://doi.org/10.1016/j.chb.2008.12.013

Berney, S., \& Bétrancourt, M. (2016). Does animation enhance learning? A metaanalysis. Computers \& Education, 101, 150-167. https://doi.org/10.1016/j.compedu.2016.06.005

Castro-Alonso, J. C., Ayres, P., \& Paas, F. (2014). Learning from observing hands in static and animated versions of non-manipulative tasks. Learning and Instruction, 34, 11-21. https://doi.org/10.1016/j.learninstruc.2014.07.005

Castro-Alonso, J. C., Ayres, P., \& Paas, F. (2015). Animations showing Lego manipulative tasks: Three potential moderators of effectiveness. Computers \& Education, 85, 1-13. https://doi.org/10.1016/j.compedu.2014.12.022

Chi, M. T. (2009). Active-constructive-interactive: A conceptual framework for differentiating learning activities. Topics in Cognitive Science, 1, 73-105. https://doi.org/10.1111/j.17568765.2008.01005.x

Chu, M., \& Kita, S. (2011). The nature of gestures' beneficial role in spatial problem solving. Journal of Experimental Psychology. General, 140, 102-116. https://doi.org/10.1037/a0021790

Cook, S. W., Mitchell, Z., \& Goldin-Meadow, S. (2008). Gesturing makes learning last. Cognition, 106, 1047-1058. https://doi.org/10.1016/j.cognition.2007.04.010

Cromley, J. G., Bergey, B. W., Fitzhugh, S., Newcombe, N., Wills, T. W., Shipley, T. F., \& Tanaka, J. C. (2013). Effects of three diagram instruction methods on transfer of diagram comprehension skills: The critical role of inference while learning. Learning and Instruction, 26, 45-58. https://doi.org/10.1016/j.learninstruc.2013.01.003

De Koning, B., \& Tabbers, H. (2011). Facilitating understanding of movements in dynamic visualizations: An embodied perspective. Educational Psychology Review, 23, 501-521. https://doi.org/ 10.1007/s10648-011-9173-8 
De Koning, B., \& Tabbers, H. (2013). Gestures in instructional animations: A helping hand to understanding non-human movements? Applied Cognitive Psychology, 27, 683-689. https://doi.org/10.1002/acp.2937

Dunlosky, J., Rawson, K. A., Marsk, E. A., Nathan, M. J., \& Willingham, D. T. (2013). Improving students' learning with effective learning techniques: Promising directions from cognitive and educational psychology. Psychological Science in the Public Interest, 14, 458. https://doi.org/10.1177/1529100612453266

Eielts, C., Pouw, W., Ouwehand, K., van Gog, T., Zwaan, R. A., \& Paas, F. (2018). Co-thought gesturing supports more complex problem solving in subjects with lower visual workingmemory capacity. Psychological Research. doi:10.1007/s00426-018-1065-9

Ekstrom, R. B., French, J. W., Harman, H. H., \& Dermen, D. (1976). Manual for kit of factorreferenced cognitive tests: Princeton: Educational Testing Service.

Engelkamp, J., \& Zimmer, H. D. (1984). Motor programme information as a separable memory unit. Psychological Research, 46, 283-299.

Glenberg, A. M. (2010). Embodiment as a unifying perspective for psychology. Wiley Interdisciplinary Reviews: Cognitive Science, 1, 586-596. https://doi.org/10.1002/wcs.55

Goldin-Meadow, S., \& Wagner, S. M. (2005). How our hands help us learn. Trends in Cognitive Sciences, 9, 234-241. https://doi.org/10.1016/j.tics.2005.03.006

Goldin-Meadow, S., Cook, S. W., \& Mitchell, Z. A. (2009). Gesturing gives children new ideas about math. Psychological Science, 20, 267-272. https://doi.org/10.1111/j.14679280.2009.02297.x

Harp, S. W., \& Mayer, R. E. (1997). The role of interest in learning from scientific text and illustrations: On the distinction between emotional interest and cognitive interest. Journal of Educational Psychology, 89, 92-102.

Hegarty, M., \& Just, M. A. (1993). Constructing mental models of machines from text and diagrams. Journal of Memory and Language, 32, 717-742.

Hegarty, M., Canham, M. S., \& Fabrikant, S. I. (2010). Thinking about the weather: how display salience and knowledge affect performance in a graphic inference task. Journal of Experimental Psychology. Learning, Memory, and Cognition, 36, 37-53. https://doi.org/10.1037/a0017683 
Höffler, T. N. (2010). Spatial ability: Its influence on learning with visualizations-a metaanalytic review. Educational Psychological Review, 22, 245-269. https://dx.doi.org/10.1007/s10648-010-9126-7

Höffler, T. N., \& Leutner, D. (2007). Instructional animation versus static pictures: A metaanalysis. Learning and Instruction, 17, 722-738. https://doi.org/10.1016/j.learninstruc.2007.09.013

Hu, F.-T., Ginns, P., \& Bobis, J. (2015). Getting the point: Tracing worked examples enhances learning. Learning and Instruction, 35, 85-93. https://doi.org/10.1016/j.learninstruc.2014.10.002

Kita, S., Alibali, M. W., \& Chu, M. (2017). How do gestures influence thinking and speaking? The gesture-for-conceptualization hypothesis. Psychological Review, 124, 245-266. https://dx.doi.org/10.1037/rev0000059

Kombartzky, U., Ploetzner, R., Schlag, S., \& Metz, B. (2010). Developing and evaluating a strategy for learning from animations. Learning and Instruction, 20, 424-433. https://doi.org/10.1016/j.learninstruc.2009.05.002

Lajevardi, N., Narang, N. S., Marcus, N., \& Ayres, P. (2017). Can mimicking gestures facilitate learning from instructional animations and static graphics? Computers \& Education, 110, 54-74. https://doi.org/10.1016/j.compedu.2017.03.010

Lakoff, G., \& Johnson, M. (1980). The metaphorical structure of the human conceptual system. Cognitive Science, 4, 195-208.

Lambourne, K., \& Tomporowski, P. (2010). The effect of exercise-induced arousal on cognitive task performance: a meta-regression analysis. Brain Research, 1341, 12-24. https://doi.org/10.1016/j.brainres.2010.03.091

Lehrl, S. (2005). Mehrfachwahl-Wortschatz-Intelligenztest MWT-B (Vol. 5). Göttingen: Hogrefe. Lindgren, R., \& Johnson-Glenberg, M. (2013). Emboldened by embodiment: Six precepts for research on embodied learning and mixed reality. Educational Researcher, 42, 445-452. https://doi.org/10.3102/0013189X13511661

Lowe, R. (2003). Animation and learning: selective processing of information in dynamic graphics. Learning and Instruction, 13, 157-176. https://doi.org/10.1016/S09594752(02)00018-X 
Lowe, R. (2004). Interrogation of a dynamic visualization during learning. Learning and Instruction, 14, 257-274. https://doi.org/10.1016/j.learninstruc.2004.06.003

Macken, L., \& Ginns, P. (2014). Pointing and tracing gestures may enhance anatomy and physiology learning. Medical Teacher, 36, 596-601. doi:10.3109/0142159X.2014.899684

Mason, L., Lowe, R., \& Tornatora, M. C. (2013). Self-generated drawings for supporting comprehension of a complex animation. Contemporary Educational Psychology. https://doi.org/10.1016/j.cedpsych.2013.04.001

McNeill, D. (1992). Hand and mind: What gestures reveal about thought. Chicago: University of Chicago Press.

Novack, M. A., \& Goldin-Meadow, S. (2017). Gesture as representational action: A paper about function. Psychonomic Bulletin and Review, 24, 652-665. https://doi.org/10.3758/s13423$016-1145-\mathrm{z}$

Pi, Z., Hong, J., \& Yang, J. (2017), Effects of the instructor's pointing gestures on learning performance in video lectures. British Journal of Educational Technology, 48, 1020-1029. https://doi.org/10.1111/bjet.12471

Ploetzner, R., \& Fillisch, B. (2017). Not the silver bullet: Learner-generated drawings make it difficult to understand broader spatiotemporal structures in complex animations. Learning and Instruction, 47, 13-24. https://doi.org/10.1016/j.learninstruc.2016.10.002

Post, L. S., van Gog, T., Paas, F., \& Zwaan, R. A. (2013). Effects of simultaneously observing and making gestures while studying grammar animations on cognitive load and learning. Computers in Human Behavior, 29, 1450-1455. https://doi.org/10.1016/j.chb.2013.01.005

Pouw, W. T. J. L., de Nooijer, J. A., van Gog, T., Zwaan, R. A., \& Paas, F. (2014). Toward a more embedded/extended perspective on the cognitive function of gestures. Frontiers in Psychology, 5:359. doi:10.3389/fpsyg.2014.00359

Renkl, A., \& Scheiter, K. (2017). Studying visual displays: How to instructionally support earning. Educational Psychology Review, 29, 599-621. https://doi.org/10.1007/s10648-0159340-4

Sanchez, C. A., \& Wiley, J. (2006). An examination of the seductive details effect in terms of working memory capacity. Memory and Cognition, 34, 344-355. 
Schmidt- Weigand, F., Kohnert, A., \& Glowalla, U. (2010). Explaining the modality and contiguity effects: New insights from investigating students' viewing behaviour. Applied Cognitive Psychology, 24, 226-237. https://doi.org/10.1002/acp.1554

Schwan, S., \& Riempp, R. (2004). The cognitive benefits of interactive videos: Learning to tie nautical knots. Learning and Instruction, 14, 293-305.

https://doi.org/10.1016/j.learninstruc.2004.06.005

Seufert, T. (2003). Supporting coherence formation in learning from multiple representations. Learning and Instruction, 13, 227-237. https://doi.org/10.1016/j.learninstruc.2004.06.005

Sheu, F. R., \& Chen, N. S. (2014). Taking a signal: A review of gesture-based computing research in education. Computers \& Education, 78, 268-277. https://doi.org/10.1016/j.compedu.2014.06.008

Stieff, M., Lira, M. E., \& Scopelitis, S. A. (2016). Gesture supports spatial thinking in STEM. Cognition and Instruction, 34, 80-99

https://doi.org/10.1080/07370008.2016.1145122

Tytler, R., Prain, V., Hubber, P., \& Waldrip, B. (Eds.). (2013). Constructing representations to learn in science. Springer Science \& Business Media.

Videler, J. J. (1993). Fish swimming. London, UK: Chapman \& Hall. https://doi.org/10.1007/97894-011-1580-3.

Wiley, J. (2019). Picture this! Effects of photographs, diagrams, animations, and sketching on learning and beliefs about learning from a geoscience text. Applied Cognitive Psychology, 33, 9-19. https://doi.org/10.1002/acp.3495

Yerkes, R. M., \& Dodson, J. D. (1908). The relation of strength of stimulus to rapidity of habit- formation. Journal of Comparative Neurology and Psychology, 18, 459-482. 


\section{Footnotes}

${ }^{1}$ The German original version of the instruction read: "Während Du Dir die Videos ansiehst, möchten wir Dich bitten, mit Deinen Händen und/oder dem Körper Gesten auszuführen. Bitte gestalte die Gesten so, dass Du das Gefühl hast, dass sie Dir dabei helfen, das Lernmaterial besser zu verstehen. Es mag vielleicht komisch klingen, aber frühere Studien haben gezeigt, dass solche Gesten das Lernen verbessern und zwar vor allem dann, wenn sie gut auf das Lernmaterial abgestimmt sind." The term 'gesture' rather than 'enactment' was used since we found participants to better understand this term.

${ }^{2}$ We checked for homogeneity of regression slopes across conditions. There were no significant interactions between spatial ability and experimental conditions for any of the dependent variables. Thus, prerequisites for treating spatial ability as a covariate were met.

${ }^{3}$ Importantly, even though medium and difficult tasks do not differ in terms of accuracy, the accuracy in more difficult tasks is achieved at higher temporal costs than that of mediumdifficult tasks. Analyzing response times for the three task difficulty levels shows a linear increase in the mean response time per item from easy tasks $(M=5705.56 \mathrm{~ms}, S E=232.78)$ to medium-difficult tasks $(M=7894.30 \mathrm{~ms}, S E=311.20)$ to difficult tasks $(M=9129.79 \mathrm{~ms}, S E=$ 336.03) with significant differences between each level (all $p \mathrm{~s}<.001$ ). 
Table 1

Means and standard deviations for students' entry characteristics as a function of experimental condition

without enactment prompt with enactment prompt

photographs video photographs video

$(n=22) \quad(n=21) \quad(n=20) \quad(n=20)$

\begin{tabular}{lllll}
\hline Spatial ability $(-10$ & $5.86(3.00)$ & $4.71(4.95)$ & $5.55(3.24)$ & $6.20(3.19)$
\end{tabular}

to +10 points)

$\begin{array}{llll}\text { Verbal ability } \quad 29.14(5.51) & 28.76(3.87) \quad 29.05(4.08) \quad 28.55(3.71)\end{array}$

(max. 37 points)

$\begin{array}{lllll}\text { Domain familiarity } & 6.61(4.64) & 6.88(5.71) & 7.13(5.56) & 6.65(5.00)\end{array}$

(max. 25 points) 
Table 2

Means and standard deviations for mental effort, number of fishes with enactment and congruency as a function of experimental condition

\begin{tabular}{lcccc}
\hline & \multicolumn{2}{c}{ without enactment prompt } & \multicolumn{2}{c}{ with enactment prompt } \\
& photographs & video & photographs & video \\
& $(n=22)$ & $(n=21)$ & $(n=20)$ & $(n=20)$ \\
\hline Mental effort & $4.98(0.95)$ & $4.08(1.73)$ & $5.00(1.49)$ & $4.83(1.17)$ \\
$(1-7)$ & & & & \\
Number of fishes & $2.00(2.73)$ & $2.33(2.76)$ & $5.90(0.45)$ & \\
with enactment & & & & \\
(max. 6) & & & & \\
Mean Enactment & $1.31(0.39)^{*}$ & $1.17(0.32)^{*}$ & $1.19(0.30)$ & \\
Congruency (min. & & & & \\
0, max. 2) & & & & \\
\end{tabular}

*Note: based on $\mathrm{n}=10$ students performing enactments in these conditions 
Table 3

Means and standard deviations for learning outcomes $(0$ - 1) as a function of experimental condition

without enactment prompt

photographs videos

$(n=22) \quad(n=21)$ with enactment prompt

photographs videos

$(n=20) \quad(n=20)$

Classification task

Easy items

$.92(.12)$

$0.93(.11)$

$0.94(.10)$

$0.93(.08)$

Medium items

$.64(.23)$

$.61(.21)$

$.76(.18)$

$.71(.22)$

Difficult items

$.64(.19)$

$.67(.19)$

$.68(.19)$

$.74(.19)$

Description task

$.69(.13)$

$.59(.19)$

$.72(.18)$

$.62(.18)$ 

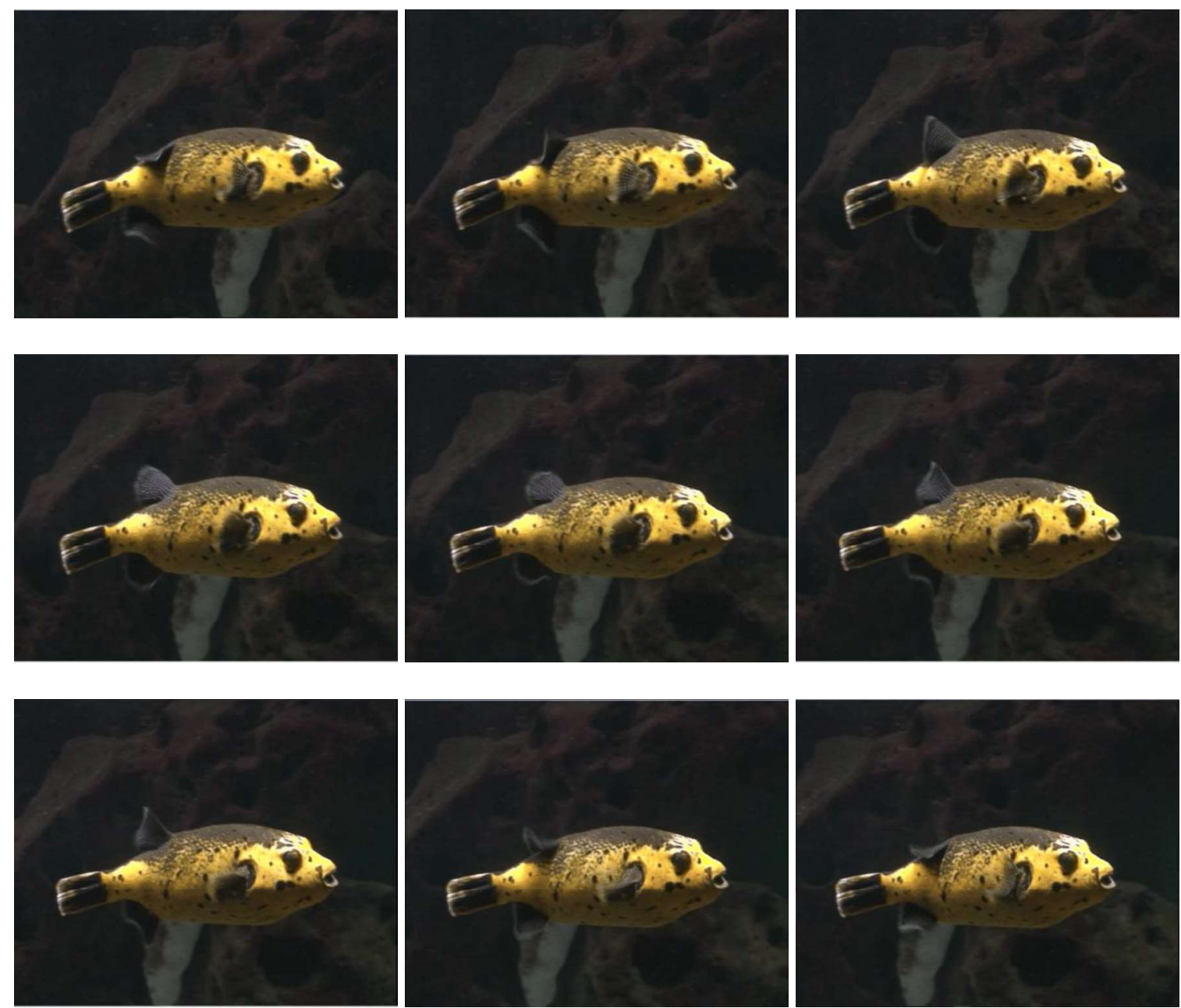

Figure 1: Sequence of stills for the tetraodontiform movement pattern (top row: 1-3, middle row: 4-6, bottom row: 5-9) 
Acknowledgement: We thank Jana Arndt for her help in conducting the study.

\section{Acknowledgements -- must be submitted separately from the manuscript \\ Acknowledgements - must be submitted separately from the manuscript}

Acknowledgement: We thank Jana Arndt for her help in conducting the study. 
- Can enacting non-human movements in your own body help learning?

- Participants studied videos or photos of fish locomotion; half were prompted to enact.

- Participants who enacted performed better on some classification tasks

- This was not influenced by the format of the visual materials

- Analysis of process measures suggests these results are due to general not specific mechanisms. 


\section{Credit Author Statement}

Katharina Scheiter: conceptualization; methodology; analysis; writing Birgit Brucker: methodology; analysis; investigation; project administration Shaaron Ainsworth: conceptualization; methodology; analysis; writing 